\title{
An Intracranial Gliosis Mimicking Neoplasm: A Dilemma
}

\author{
Zhi-hong Shao, ${ }^{1}$ Guo-liang Wang, ${ }^{1}$ Xiang-hua Yi, ${ }^{2}$ and Pei-jun Wang, ${ }^{1, *}$ \\ ${ }^{1}$ Department of Radiology, Tongji Hospital of Tongji University, School of Medicine, Shanghai, China \\ ${ }^{2}$ Department of Pathology, Tongji Hospital of Tongji University, School of Medicine, Shanghai, China \\ "Corresponding author: Pei-jun Wang, Department of Radiology, Tongji Hospital of Tongji University, School of Medicine, Shanghai 200065, China. Tel: +86-2166111206, Fax: \\ +86-2156952231, E-mail: tongjipjwang@sohu.com
}

Received 2013 December 11; Revised 2014 August 16; Accepted 2014 October 13.

\begin{abstract}
Intracranial gliosis has no typical clinical signals or imaging characteristics. Therefore, it can be easily misdiagnosed as neoplasm. Hereby, we report a unique case of gliosis that grew outward from the surface of the brain. MRI depicted its signal and enhancement pattern similar to the cerebral gray matter. The diagnosis was confirmed by pathology and immunohistochemistry. Although it was difficult to reach a diagnosis, correlating its origin, growing pattern and MR features and knowing that gliosis can present this way may help in differentiating it from other diseases.
\end{abstract}

Keywords: Gliosis, Pathology, Magnetic Resonance Imaging (MRI), Neoplasm

\section{Introduction}

Intracranial gliosis is an uncommon disease rarely reported and can be easily misdiagnosed as neoplasm. There can be significant overlap in the radiologic presentation between intracranial gliosis and other neoplastic or nonneoplastic diseases including glioma, demyelinating disease, infection, and inflammation. However, here we report a unique case of intracranial gliosis with specific MR appearances that presented as a localized mass arising from the surface of the brain. It demonstrated signal and enhanced pattern similar to the brain parenchyma, which rendered the differential diagnosis of low-grade astrocytoma and meningioma difficult. To the best of our knowledge, intracranial gliosis presenting in this way has not been reported previously.

\section{Case Presentation}

A 40-year-old man complained of dizziness, and palpitation for one year. The blood pressure was 180/160 $\mathrm{mmHg}$, and decreased to $150 / 95 \mathrm{mmHg}$ after treatment with oral antihypertensive drugs at the local hospital about six months ago. However, there was no significant improvement in his symptoms and his headache aggravated. The patient had a history of craniofacial trauma for which he was treated conservatively about 25 years ago.

MRI (3.0 Tesla, Verio, Siemens Medical Solution) of the head revealed a localized mass at the bottom of the brain. The maximum dimensions of the entire mass were $5.2 \mathrm{~cm} \times 6.1 \mathrm{~cm}$. The mass showed homogeneous isointense-signal-intensity on $\mathrm{T} 1$ and T2-weighted images (Figure $1 \mathrm{~A}$ and $\mathrm{B}$ ) with no apparent contrast enhancement on gadolinium-enhanced T1-weighted image (Figure $1 C$ ) that was consistent with the cerebral gray matter. It appeared as a well-defined mass except for the absence of clear demarcation from the anterior perforated substance of the brain and a pedunculated attachment on coronal and sagittal T2-weighted images (Figure 1D and E). Vesselscerebrospinal fluid interface was noticed around the lesion except for the side of pedunculated attachment, just like an extra-axial neoplasm such as meningioma. The mass extended to the base of the frontal and middle cranial fossa. The "cleft sign" could be observed between the lesion and the inner table of the skull. Mass effect was noted on the adjacent gyri, flow void vessels were displaced, and no surrounding edema and meningeal enhancement or the dural tail sign was seen.

The intracranial mass was removed surgically. The mass actually aroused from the surface of the brain parenchyma. On the cut surface, the mass appeared as a fairly well defined, gray-white, soft, hypovascular, unencapsulated solid mass. Histopathologically, the increased and evenly distributed cellularity was demonstrated in some background neurons from the gray matter (Figure $1 F)$. Cellular pleomorphism or mitotic figure was not apparent. Immunohistochemically, glial fibrillary acidic protein (GFAP) stain showed astrocytes with dense staining of slightly enlarged cell bodies and highlighted cell processes (Figure 1G). The increased cellularity was positive for S-100 and negative for epithelial membrane antigen (EMA), Vim, CD34, cytokeratin (CK), Ki-67 (Figure 1H), and P53. Based on 

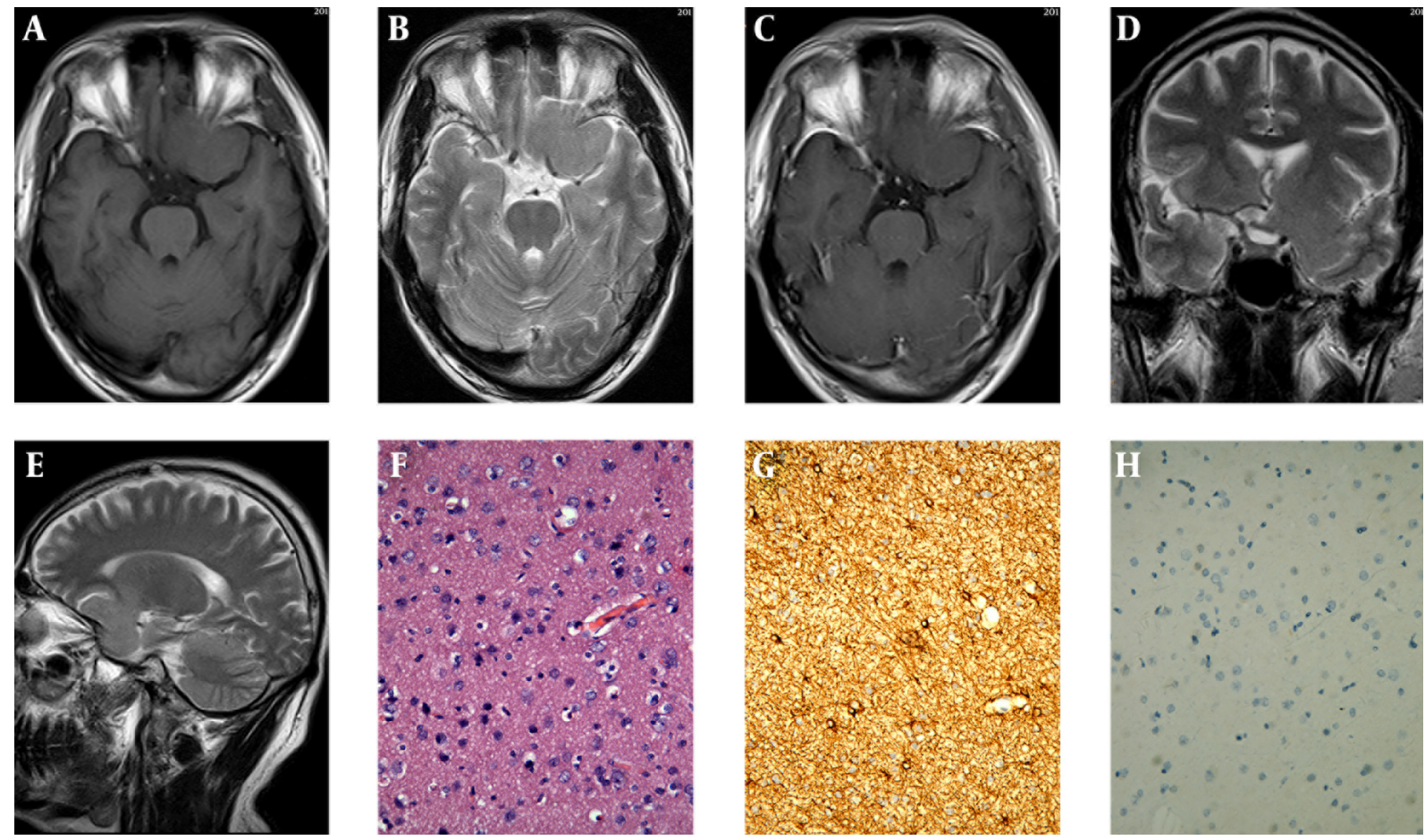

Figure 1. MRI of an intracranial gliosis in a 40-year-old male; A and B, Shows a localized mass at the anterior perforated substance with similar signal to the cerebral gray matter on T1- and T2-weighted images; C, No apparent contrast enhancement on gadolinium-enhanced T1-weighted image; D and E, The mass appears to be absent of clear demarcation from the parenchyma of the brain and with a pedunculated attachment on coronal and sagittal T2-weighted images; $\mathrm{F}$, The increased and evenly distributed cellularity is demonstrated in some background neurons from gray matter (Hematoxylin and Eosin staining, $\times 200$ ); G, GFAP stain shows astrocytes with the dense staining of slightly enlarged cell bodies and the highlighted cell processes; $H$, The increased cellularity is negative for Ki-67 (immunohistochemistry, $\times 200$ ).

these pathological findings, we considered this mass to be intracranial gliosis.

The patient was relieved from headache and other symptoms after the surgery, and remained clinically well with no lesions detected for a follow-up period of one year.

\section{Discussion}

Intracranial gliosis as an uncommon non-neoplastic disease is the brain's way of reacting to injury and insult. It predominantly results from proliferation of astrocytes (1-4). Intracranial gliosis typically manifests in children or young adults, and may involve both gray and white matter, especially in the frontal and temporal lobe. The lesion in this report that originated from the gray matter of the anterior perforated substance of brain may be related to the history of the craniofacial trauma that happened 25 years ago.

Intracranial gliosis is a non-specific change that can clinically and radiologically mimic a neoplastic or nonneoplastic mass lesion. It is even common to observe at least some degree of reactive astrocytosis adjacent to or associated with these conditions. Therefore, the distinction between gliosis and other mass lesions is often the most difficult challenge of diagnostic radiology, even neuropathology, especially for the routine evaluation of small biopsy specimens (5-7).

MRI is still of critical importance for gliosis. On MRI, intracranial gliosis, similar to inflammation or low-grade astrocytoma, is usually depicted as an ill-defined margin mass with slight hypo- or isointense signal on T1weighted images, hyperintense signal on T2-weighted images, nonenhancing or patchy, linear enhancement, and no obvious or minimal mass effect and perilesional brain edema (8). The lesion has no calcification or cystic change. The MRI appearances of intracranial gliosis and inflammation are alike somewhat depending on infiltration of inflammatory cells in the area of gliosis. The lesion enhancement results from variable breakdown of the blood-brain barrier and abnormality of vascular permeability. However, the lesion in this report showed the same isointense signal as the gray matter on T2-weighted image, no hyperintense signal as inflammation, low-grade astrocytoma or other intra-axial lesions, and no enhancement, which 
may be related to the absence of inflammatory cell infiltration and integrity of vascular permeability. Moreover, low-grade astrocytoma often occurs in or near the corticomedullary junction, and is seldom located in the surface of the brain parenchyma and becomes mass like an extraaxial lesion. Thirdly, the gliosis we reported is also easily misdiagnosed as meningioma due to its isointense signal on T1 and T2-weighted images, and cerebrospinal fluid interface around the lesion; whereas, the mass attachment to the brain base with a pedunculation on coronal and sagittal T2-weighted image points towards an intra-axial lesion. A typical meningioma is a markedly enhanced lesion with a broad base of dural attachment, and a dural tail is often seen. However, the lesion in this report shows no apparent contrast enhancement or meningeal enhancement on gadolinium-enhanced T1-weighted image. Magnetic resonance spectroscopy may be helpful for the differential diagnosis of gliosis.

The diagnosis of intracranial gliosis depends on its histopathology analysis and the differential diagnosis should be made from low-grade astrocytoma $(6,7)$. Microscopically, similar to low-grade astrocytoma, gliosis may result in increased cellularity. The increased cellularity associated with gliosis is generally evenly distributed, but astrocytoma is not. The differences between gliosis and astrocytoma may not be readily apparent on routine hematoxylin eosin staining and may require immunohistochemical stains to be clearly visualized. The most commonly used markers to differentiate them are GFAP, proliferation markers (e.g. Ki-67) and p53. Gliosis has a feature of evenly spaced astrocytes with multiple, thin long radiating glial processes. In contrast, astrocytic cells of astrocytoma cluster and have shorter and thicker processes. Gliosis usually has a lower proliferation index than astrocytoma for Ki-67. Astrocytoma usually has strong and diffuse p53 staining; however, gliosis may show negative. Furthermore, the distinction between gliosis and low-grade astrocytoma is often the most difficult challenge, even neuropathologically. Several studies demonstrated IDH1-specific immunohistochemistry may be useful for further differential diagnosis, but a negative IDH1 immunostain does not exclude an infiltrating glioma.

Intracranial gliosis may not necessarily require surgery. For lesion of uncertain diagnosis or difficult to distinguish from glioma, stereotactic biopsy is feasible. Most of the patients with gliosis have good prognosis, and long-term survival.

In summary, intracranial gliosis is easily misdiagnosed for other neoplastic or non-neoplastic lesions. The age of the patient, a previous history of disease relating to the brain especially radiation therapy or trauma, location and the radiologic presentation of the lesion, are all important and very useful information. The case we reported has a unique feature of origin, growing pattern and MR signals. Bearing in mind that gliosis can present this way may be useful in differentiating it from other diseases.

\section{Acknowledgments}

We would like to thank Dr. Sewraj-Fagoonee for her assistance in revising the manuscript.

\section{Footnote}

Authors' Contribution: Zhi-hong Shao was responsible for drafting of the article and revising it critically for important intellectual content. Guo-liang Wang carried out acquisition and analysis of clinical and image data. Xianghua Yi performed analysis and interpretation of pathological data. Pei-jun Wang was responsible for final approval of the submitted version.

\section{References}

1. Hwang SY, Jung JS, Kim TH, Lim SJ, Oh ES, Kim JY, et al. Ionizing radiation induces astrocyte gliosis through microglia activation. Neurobiol Dis. 2006;21(3):457-67. doi:10.1016/j.nbd.2005.08.006. [PubMed:16202616].

2. Faulkner JR, Herrmann JE, Woo MJ, Tansey KE, Doan NB, Sofroniew MV. Reactive astrocytes protect tissue and preserve function after spinal cord injury. J Neurosci. 2004;24(9):2143-55. doi: 10.1523/JNEUROSCI.3547-03.2004. [PubMed: 14999065].

3. Wasserman JK, Yang H, Schlichter LC. Glial responses, neuron death and lesion resolution after intracerebral hemorrhage in young vs. aged rats. Eur J Neurosci. 2008;28(7):1316-28. doi: 10.1111/j.14609568.2008.06442.x. [PubMed:18973558].

4. Buffo A, Rite I, Tripathi P, Lepier A, Colak D, Horn AP, et al. Origin and progeny of reactive gliosis: A source of multipotent cells in the injured brain. Proc Natl Acad Sci U S A. 2008;105(9):3581-6. doi: 10.1073/pnas.0709002105. [PubMed: 18299565].

5. Capper D, Sahm F, Hartmann C, Meyermann R, von Deimling A, Schittenhelm J. Application of mutant IDH1 antibody to differentiate diffuse glioma from nonneoplastic central nervous system lesions and therapy-induced changes. Am J Surg Pathol. 2010;34(8):1199-204. doi: 10.1097/PAS.ob013e3181e7740d. [PubMed: 20661018].

6. Camelo-Piragua S, Jansen M, Ganguly A, Kim JC, Louis DN, Nutt CL. Mutant IDH1-specific immunohistochemistry distinguishes diffuse astrocytoma from astrocytosis. Acta Neuropathol. 2010;119(4):509-11. doi: 10.1007/s00401-009-0632-y. [PubMed: 20044756].

7. Rivera-Zengotita M, Yachnis AT. Gliosis versus glioma?: don't grade until you know. Adv Anat Pathol. 2012;19(4):239-49. doi: 10.1097/PAP.ob013e31825c6a04. [PubMed: 22692287].

8. Lee D, Thaler JP, Berkseth KE, Melhorn SJ, Schwartz MW, Schur EA. Longer $\mathrm{T}(2)$ relaxation time is a marker of hypothalamic gliosis in mice with diet-induced obesity. Am J Physiol Endocrinol Metab. 2013;304(11):E1245-50. doi: 10.1152/ajpendo.00020.2013. [PubMed: 23548614]. 